\title{
12. Research as an information systems domain
}

\section{Craig McDonald, School of Information Sciences and Engin- eering, University of Canberra}

\begin{abstract}
There is growing interest in the use of ICT in the domains of science and research. Little of that interest is currently focused on the systems aspects of those domains, but instead looks piecemeal at the way they may make use of particular ICT tools. Two important opportunities exist here for the information systems discipline; firstly, to analyse it as a human activity system to see how ICT can be systematically employed in its activities, and secondly, to view the deployment and adoption in society of knowledge created by research as a matter of information systems inter-operation. This paper looks at how the foundation ideas of IS might be mobilised to improve the vital domain of research.
\end{abstract}

\section{Introduction}

Information systems is a discipline that interests itself in the interaction of information technologies with human activity systems. The purpose of this paper is to examine some aspects of research as a human activity system and the role information systems might play in it.

\section{e-Science and e-Research}

Both the Chair of the ARC and Australia's Chief Scientist have spoken recently about eScience and e-Research. Cram's (2003) 'A Roadmap for e-Research' and Batterham's (2003) 'E-Science: A Frontier Technology for Achieving the National Research Priorities' set the scene for the section on e-Science in the 'Smart Use of Information Technology Systems' (SUITS) bid. Both of these sources, from peak government advisors, emphasise the use of information and communication technologies (ICT) in the way research and innovation will be conducted in the future.

The terms e-Science and e-Research are not well differentiated. E-Science is usually understood to be related to the use of ICT in scientific research, particularly that needing high computing power and vast data sources in a highly distributed grid environment (e.g. the National E-Science Center). Typical domains include astronomy, physics, geology, and so on. The Australian Partnership for Advanced Computing (APAC), Australasian Workshop on Grid Computing and e-Research (AusGrid) and, presumably, National ICT Australia (NICTA), are the kinds of organisations involved in e-Science. The technologies they are developing and promoting include broadband, middleware, repositories of scientific data, sensors and instrumentation, distributed computational power, and so on.

The UK e-Science Grid conceives of:

an e-Scientist's workbench ... [that] aims to support: the scientific process of experimental investigation, evidence accumulation and result assimilation; 
the scientist's use of the community's information; and scientific collaboration, allowing dynamic groupings to tackle emergent research problems.

E-Research is a broader term that, Cram (2003) says, 'concerns the ways that Researchers, Research Students, Scholars and Entrepreneurs use and will use Information and Communications Technologies (ICT)' in the context of innovation and knowledge application'. He argues 'Research is to Innovation as Sunlight is to Photosynthesis'. The first theme of this paper, then, is: recognising research as a systematic, human activity that can make use of the 'big ICT' envisaged in e-Science

In a major Australian Department of Education, Science and Training (DEST) study, 'Changing Research Practices in the Digital Information and Communication Environment', released in late 2003, Prof. John W. Horton has reviewed the current and future directions for research. The changing nature of research, who does it, how it is funded, what research practices are in use and the role of ICT are discussed at length, and key findings about the systems needed to underlie research in the future are presented.

To an information systems person, however, Horton is trapped in a traditional view of knowledge. While his study covers knowledge production and its dissemination in documents, it does not cover its use. His view is that ICT can contribute to collaboration between researchers' access to knowledge as part of the production process and the publishing of knowledge. But what of its deployment and use to achieve human ends? Consequently, the second theme of this paper is: recognising the use of research, not just its production.

For the reasons underlying the two themes of this paper, research is a suitable domain for IS attention. This paper will use the term e-Research to mean IS work in the research domain.

\section{Information systems}

Information Systems (IS) is an active, interventionist discipline that mobilises information and knowledge so people can effectively take knowledgeable, informed actions in their organisational and social setting. It is concerned with understanding and formalising areas of human activity and developing IT-based systems that responsibly intervene in those areas for the benefit of all stakeholders. The shape of IS practice is a bit like the middle loop in Figure 12.1: analysis, modelling, systems construction and intervention in a human activity system.

Figure 12.1. A general model of information systems activity.

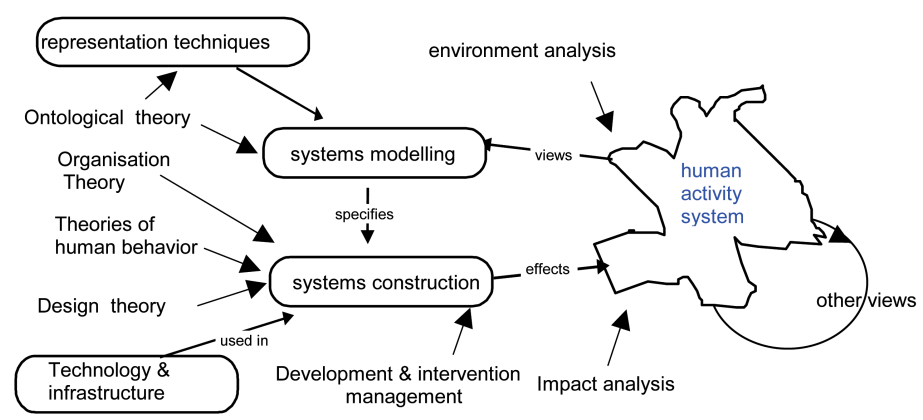

The outer components in the diagram show some of the generic theories, tools and techniques used in the process of IS work. The theories and understandings from onto- 
logy, organisational behaviour, ethics, and so on inform IS practice. Information technology and infrastructure are a driving force in the IS process, having a particular effect on representational techniques and the systems specification possibilities.

Looked at from an IS practice perspective, research is a human activity and the research world a human activity system. As such, it is susceptible to IS intervention. Just as we have e-business, e-learning or health informatics, so we can have e-research.

Using this model, we can examine research as a human activity system, and look at the current technologies in use.

\section{Research, human activity systems, and ICT}

A human activity system can be analysed at different levels of granularity, from the most fine-grained, personal level to the societal level.

Research at the personal level involves issues of motivation, personality, knowledge and skill that the individual researcher brings to their work. The kinds of activities researchers undertake include literature work, research design, data collection and analysis and research reporting. The technologies they use are:

1. document management technologies, which are used for document access and retrieval (the Web, library databases), document tracking (Endnote and Procite), and document generation and publication;

2. data collection and management tools, including data loggers of various kinds, Web surveys and focus groups, data mining and the recording of laboratory records in image, text, recordings and video;

3. analytic tools, for dealing with quantitative data (SPSS) and qualitative content analysis (NVivo and Leximancer), and visualisation and simulation software, which are examples of special purpose technologies used in particular kinds of projects.

The social level of human activity consists of the personal networks, the public behaviours, norms and culture that are exhibited by research groups and collaborations. These are supported by communication technologies, email, videoconferencing and collaborative tools such as Sharepoint, Yahoo and CommunityZero.

The organisational level of human activity systems concerns the processes, accountability and power structures in organisations such as universities, the Commonwealth Scientific and Industrial Research Organisation (CSIRO), Defense and parts of industry. Typically, ICT infrastructure is owned at this level. Systems like ResearchMaster are well established in universities to help manage the flow of research projects, publications, and so on, but such systems are probably not part of the research human activity system as they are not concerned with research content.

The societal level addresses questions about who pays for, and who benefits from, research.

These levels are not hierarchical. For example, a social network exists independently of organisations and societies. Nor is it necessary that human activity systems are purposeful. In fact, from an IS perspective, we are often more interested in the 'metabolism' of these systems than their justification. The ways people act are what IS contributes to, and social, political and cultural factors are always active in all human activity systems (Checkland and Scholes, 1990) .

As demonstrated above, the research human activity system, like all data, information and knowledge systems, can make effective piecemeal use of information technologies. 
ICT is most active at the personal level, with the adoption of tools for individual tasks. Research infrastructure is a feature of the organisational level where there is finance and ongoing structures to manage the assets. Technology is an instrumental component of the IS approach to human activity systems; one that offers opportunities and limitations. At the foundation of the IS approach, however, are the very significant human, social and use aspects of technologies, and IS as a discipline has the role of systematising a range of ICT breakthroughs that, combined, can address a problem more effectively than can individual technologies. In the research human activity system there is plenty of technology push, but little systems-pull.

\section{Information systems in research}

The possibilities of systems-pull (or, inversely, the problem of 'systems failures') in the research domain are rampant. The fact that data sets are typically not warehoused and re-used is an example of failure at the data level.

At the information level, McDonald (2003) argued that current methods of organising and mobilising this research are flawed. Considered as a whole, the applied science literature is:

1. Dispersed: It is scattered across different kinds of literature such as books, periodicals, research papers, technical reports, proceedings, which are located all over the globe. It is possible that research is unwittingly being duplicated because the original was not found in the literature review.

2. Dated: Some knowledge, created long ago, has been superseded by more recent work but still remains in the literature with a corresponding potential to mislead.

3. Underutilised: Studies indicate that no more than 20 per cent of the knowledge available in research institutes is really being put to use. Therefore, the full weight of current human knowledge is not being brought to bear on problem solving.

4. Expanding rapidly: The quantity of knowledge is increasing at an exponential rate.

5. Variable in quality: The reliability of public knowledge is complex. 'Textbook Science' is more reliable than primary (e.g. research papers) and secondary literature (e.g. review articles). Furthermore, knowledge that is reliable in one context may not be so reliable in another.

6. Inconsistent: Considerable contradictions have been found within published knowledge, and between the published knowledge and expert opinion.

7. Incomplete: There are considerable gaps in published knowledge.

8. Slow to be published and applied: The path from applied science research to decision making in the field can be long and inefficient. Publication in scientific journals can take 12 to 18 months after acceptance, which itself may have taken a year to achieve.

Clearly, there is a large knowledge management problem to be addressed here, even if the information management systems (document collection, indexing, bibliographic and full-text databases that store and deliver papers) were effective. We are stuck in a very outmoded system that serves neither researchers nor practitioners adequately.

There are attempts to address these problems. The Cochrane Collaboration has successfully adopted 'systematic reviews' or meta-analyses as a method for getting the best scientific results to practitioners and other researchers. Meyers, in the Communications of the AIS has papers that are regularly revised, and WIKI systems allow multiple people to continuously contribute to and revise a paper. 
An IS approach to these problems would use a variety of technologies and methods, but IS theories, tools and techniques will need to be deployed, reviewed and, probably, new IS approaches developed. Some parts of an IS approach to e-Research might be:

1. research data warehouses;

2. ontological systems for content organisation;

3. meta-analysis to bring together work with a similar ontological basis;

4. more advanced techniques of domain analysis;

5. knowledge management mechanisms for evidence-based research;

6. serious e-libraries (see DSpace);

7. development of domain-specific patterns.

It is at the knowledge level, that e-research may well have its greatest impact. Knowledge management systems (KMS) technologies may be at the heart of a new kind of system. This system would be charged with representing the knowledge reported in a domain of research and, through a set of interface systems, employ the knowledge base in different ways to meet some of the needs in a range of human activity systems. For example, a decision support system would use the KMS as a model of a domain to allow scenario processing; an expert system would give advice using the KMS as a knowledge base and justify the advice on the basis of the publications from which the KMS has been built; a Computer Aided Instruction (CAI) interface would allow the KMS to form the basis of courses in the domain; researchers and research bodies could use the KMS as a source for literature reviews and hypothesis testing. Each of these interface systems would have specific systems components suitable to their purposes but would rely on the core KMS as the source for their domain knowledge. The KMS would be self-maintaining as each new research report that became available would be represented as a new documentrelated knowledge base and so participate immediately in the various uses to which the system is being put. Such a system would be domain specific, rather like the 'specialist libraries' of the past. The various needs of the different stakeholders could be met from a single core of knowledge.

Proposals like this are not new. A century ago, Paul Otlet was presenting a similar notion (see various papers by W. Boyd Rayward). We may now, however, be in a position to bring new technologies to bear on e-Research, but only if IS takes a major role in the intervention that such technology might make to the research human activity system. Without IS, another technology failure would most likely be imminent.

\section{Research and practice interoperability}

The KMS described above introduces the second e-Research issue that IS needs to address - the interaction of the research and practice domains.

The idea that research results need to be socially useful is not new either. The nature of knowledge, its production and use, has long been a topic of debate and academic research. In Australia, research has been largely a publicly funded activity, and government is now casting an increasingly critical eye over the way it is currently performed. DEST's research network initiative (the context in which this paper is written) is the latest in a series of moves to promote interdisciplinary research that aims to create and apply knowledge to address problems of national significance. Ronayne (1997) put it this way:

In Mode 1 problems are set and solved in a context governed by the interests of a largely academic community. By contrast, Mode 2 knowledge production is carried out within the context of application. It is intended to be useful to 
someone other than specifically the practitioner, be this industry, government or society generally; and this requirement is present from the beginning.

Batterham (2003) describes Mode 1 as 'discipline based; distinguishes between theoretical core and its conversion to application' while Mode 2 is 'multidisciplinary, team based; Constant flow between basic \& applied; Discovery occurs where knowledge is developed and put to use'. The search for integration between research and practice is a priority.

But these are two very different worlds - human activity systems that share knowledge, but not purpose, method or people. The relationship has normally been one of knowledge provision on one side and adoption on the other. There are many examples of effective interoperation projects (for example, in most of Australia's Cooperative Research Centres).

In this way of thinking, the relationship between research and practice is like that between two organisations engaged in e-Commerce. The currency is knowledge, with practice providing relevance and raw data to theory, and research providing economically useful, causally based knowledge and interventions to practice. Figure 12.2 shows two information systems planes, one for research, the other for practice, with the suggestion of a third interoperation system in between.

Figure 12.2. Traditional interoperation of research and practice systems.

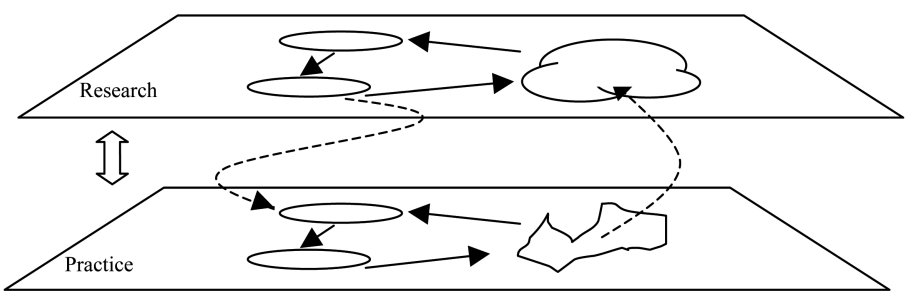

E-Commerce, like interoperation between systems, has become a significant part of IS. Recent work in terms of ontologies and the Web (Moody, 2000) and in object orientation, agents, XML, and so on, is accelerating the field. So patterns to support this kind of relationship between research and practice already exist.

But the KMS described earlier implies a different view. It concentrates not on the trading between systems but on sharing what they have in common. It suggests a deeper association of human activity systems, not just ICT mediated communication.

\section{Conclusion}

From an IS research perspective the research domain presents an opportunity to develop and test new ideas in IS. Our challenge is to make a serious effort to create systems architectures, define and mobilise technologies and specify processes that address e-Research.

ICT does not work effectively in human activity systems without IS. It can work at the individual and social levels, but not at the organisational or societal levels. So there is a demand and supply relationship between ICT and IS. From the IS perspective, new ICT offers opportunities for building new types of systems, and it can be argued that IS has been slow to adopt some of these technologies. However, IS has certainly been slow in demanding that ICT produce tools for solving new types of problem. Looking at research as an IS domain challenges us to think of, and to start driving, the next steps in informatics development. 
This paper has looked at IS and its application to the research domain. Some IS foundations this review has exposed are: ICT as tools verses ICT as instruments in systems; aspects of human activity systems as they present in research; the need for integration of systems that address data, information and knowledge; and an approach to integrating research and practice.

The way forward would be to select a small research area, see it as a human activity system and knowledge domain, look to understanding and formalising it in IS terms, and developing IT-based systems that responsibly intervene in it for the benefit of all stakeholders. 